Мамедова Г.А., Агаев Ф.Т., Зейналова Л.А.

Институт Информационных Технологий НАНА, Баку, Азербайджан

depart10@iit.ab.az

\title{
ИСПОЛЬЗОВАНИЕ СОЦИАЛЬНЫХ СЕТЕЙ ДЛЯ ПЕРСОНАЛИЗАЦИИ ЭЛЕКТРОННОГО ОБРАЗОВАНИЯ
}

В настоящее время современные социальные технологии используют сотни миллионов пользователей, они доступны бесплатно, привлекательны и интересны. $B$ статье рассматриваются возможности использования социальных сетей для улучшения электронного образования высшего учебного заведения. Учитывая большой объем информации, распространяемьй студентами вузов в социальной сети, в статье для персонализации контента учебных материалов предлагается использовать методы кластеризачии данных - k-теапs (к-средних). Полученные результаты исследования могут быть использованы педагогами и инструкторами высшего учебного заведения для улучшения содержания электронного курса и персонализащии электронного образования.

Ключевые слова: сочиальная сеть, электронное образование, персонализация учебного контента, методы кластеризачии.

\section{Введение}

Научные достижения в области информационных технологий привели в начале XXI века к развитию концепции персонального образования (англ. PLE - Personal Learning), идея которой состояла в том, чтобы обеспечить педагогов и кураторов электронных курсов возможностями настраивать обучение под потребности каждого, отдельно взятого обучаемого. Понятие «персонализация образования» шире, чем просто «индивидуализация» или «дифференциация», поскольку оно дает обучаемому определенный выбор того, что изучается, когда оно изучается и как оно изучается.

Персонализированное обучение - это адаптация педагогики, учебной программы и учебных сред для удовлетворения потребностей и стилей обучения отдельно взятого обучаемого. Ключевыми элементами, которые настраиваются в персонализированном электронном обучении, являются: темпы обучения, учебный подход, обучающие действия, образовательный контент. В действительно персонализированной среде электронного обучения студентам предоставляется возможность узнать, чего они хотят, когда захотят, и даже метод обучения [1].

Персонализация образования непосредственно связана с потребностью перехода к обществу знаний, когда в центре образовательного процесса стоит обучающийся и обучение осуществляется по месту его нахождения, а образовательная среда настраивается под него.

Многие зарубежные вузы, осознав это, приступили к созданию систем управления образованием (англ. LMS - Learning Management System), позволяющих персонализировать образовательные среды [2], такие, как Болтонский, Манчестерский университеты в Англии.

Фактически речь идет уже не об индивидуализации образования, а о персональном образовании, формировании персонального образовательного пространства (т.е. фактическом создании «киберличности» в образовательном пространстве). Это в полной мере позволяет учитывать персональные психофизиологические характеристики обучающегося: от темпа усвоения знаний до генетических характеристик.

В настоящее время по всему миру принято использовать в университетах системы управления обучением. Однако эти платформы используются, главным образом, для административных целей, и их образовательный эффект имеет мало значения. В таких системах для образовательной перспективы у студентов есть возможность проверить учебный контент, и они могут взаимодействовать со своими одноклассниками и учителями. 
Однако жесткая структура общих систем управления электронным обучением не стимулирует активное участие обучаемого в учебном процессе и совершенствовании его знаний.

Понимание этого привело в первом десятилетии XXI века к многочисленным попыткам создания такой среды обучения, в которой образовательная среда обучаемого была бы соединена с персональными пространствами других обучаемых для эффективного обмена знаниями и совместного создания новых знаний. Использование социальных приложений и социальных сетей обеспечивает иной подход к электронному обучению, чем тот, который обеспечивается использованием систем управления обучением.

Социальная сеть расширяет индивидуальные потребности студента в процессе обучения, а также обеспечивает контакт с большим количеством людей, разделяющих одни и те же интересы и цели. Поэтому в данной статье исследуются возможности использования социальных сетей для улучшения электронного образования путем персонализации контента учебных материалов. Для выявления схожих по содержанию учебных материалов, распространяемых студентами в социальной сети, используется метод кластеризации данных k-means (к-средних).

\section{Отличительные особенности стандартных систем управления образованием и систем с использованием социальных сетей}

Хотя большинство социальных сетей, которые в настоящее время существуют, разрабатывались не для образовательных целей, у них есть определенные функции, которые могут оказаться очень интересными и важными в образовательном процессе. В них существуют некоторые инструменты для поддержки электронного обучения, включающие блоги, форумы для обсуждения, чаты, совместное использование файлов, видеоконференции, электронные портфолио и вики. Все они могут использоваться для поддержки действий, связанных с процессом обучения. Некоторые из этих инструментов можно интегрировать в платформы для электронного обучения. На рисунке 1 представлена схема совместной учебной платформы с использованием социальной сети.

Например, блоги социальных сетей могут использоваться студентами для распространения своих идей, публикации своих работ, а закладки позволяют им добавлять гиперссылки для просмотра определенных сайтов учащимися с аналогичными интересами. Для размещения мультимедийной информации можно использовать сеть обмена файлами - YouTube.

Социальные технологии могут использоваться студентами для совместного изучения способов решения своих проблем с членами группы или для организации совместных учебных и обучающих групп. Сотрудничая со сверстниками, они расширяют свои формальные и неформальные взаимодействия, устанавливают с ними активную связь, стремятся работать с конкретными результатами или производить результаты в онлайн- и автономном режимах.

В публикациях некоторых исследователей обсуждаются возможности широкого использования социальных технологий в области высшего образования [3-6]. Некоторые авторы даже утверждают, что социальные технологии поддерживают конструктивистские подходы к обучению и дают возможность обмениваться онлайн-обучением в большей степени, чем это было в традиционных учебных средах [7]. 


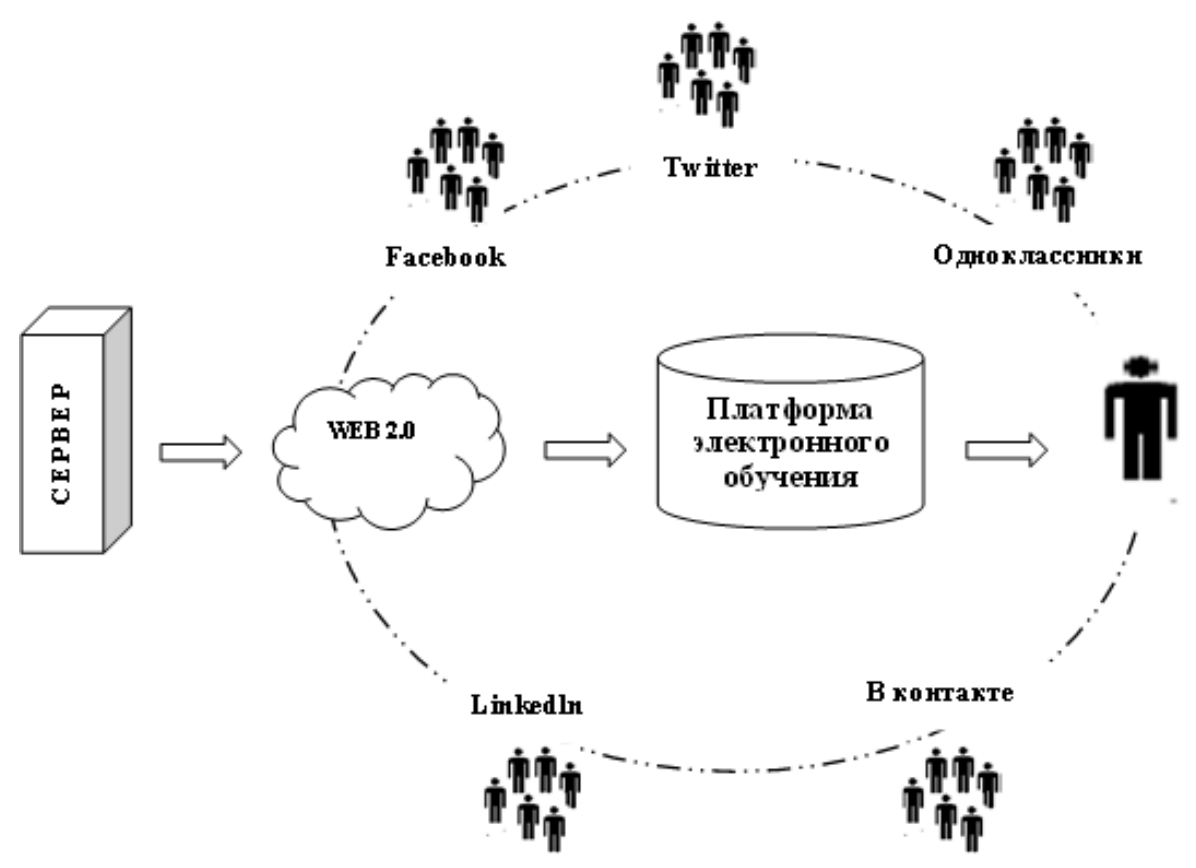

Рис.1. Совместная с онлайн-социальными сетями среда электронного обучения

Социальные технологии поддерживают гибкость в процессах обучения и обеспечивают легкую публикацию, обмен идеями и повторное использование содержания исследования и комментариев. Они также поддерживают ссылки на соответствующие ресурсы в информационной среде, которыми управляют сами студенты и лекторы. Некоторые авторы утверждают, что образовательная деятельность в социальных сетях ценна для развития навыков совместной работы студентов, где студенты используют привлекательные для них социальные технологии [8], а в некоторых случаях использование социальных сетей способствовало более высоким показателям в учебной деятельности [9].

Благодаря использованию социальных технологий, таких, как блоги, вики-сайты и сайты социальных сетей, студенты чаще становятся более активными, более независимо выражают себя, поскольку им предоставляется свобода публиковать свою работу в Интернете через блоги и вики. Использование социальных технологий может еще больше повысить мотивацию студентов и привлечь внимание к деталям, что приведет к общему улучшению качества работы. Авторы отметили, что когда студент публикует свои работы в социальной сети, они, в основном, оригинальны, интересны и привлекательны для других, позитивно оцениваются сверстниками и преподавателями.

Использование социальных сетей для поддержки электронного обучения является частью активного и социального типа образования, в котором студенты ориентированы на решение проблем. Основными моментами процесса обучения являются саморегулируемые действия по решению проблем, что предполагает подход к социальному конструктивизму, в котором учащийся должен управлять своим собственным процессом обучения. Этот тип обучения означает, что невозможно структурировать и заранее определять действия учащегося в процессе обучения. Действия должны начинаться с проблемы или проекта, а сеть, в свою очередь, предоставляет инструменты и ресурсы, поддерживающие учащегося в процессе решения проблемы. В таблице 1 показаны отличительные особенности платформ электронного обучения и с использованием социальных сетей [10]. 


\section{Таблица 1.}

Отличительные особенности платформ электронного обучения и социальных сетей

\begin{tabular}{|c|c|}
\hline Платформа электронного обучения & Социальная сеть \\
\hline $\begin{array}{l}\text { Ограниченная среда для пользователей } \\
\text { платформы }\end{array}$ & $\begin{array}{l}\text { Она охватывает всю сеть друзей и друзей его } \\
\text { друзей в зависимости от степени } \\
\text { конфиденциальности }\end{array}$ \\
\hline $\begin{array}{l}\text { Студент играет пассивную роль. Он только } \\
\text { может получить доступ к материалу, } \\
\text { предоставленному преподавателем }\end{array}$ & $\begin{array}{l}\text { Студент играет активную роль. Принимает } \\
\text { участие в формировании процессов обучения }\end{array}$ \\
\hline $\begin{array}{l}\text { Содержание разработано преподавателем и } \\
\text { используется обучаемым }\end{array}$ & $\begin{array}{l}\text { Студент сам может создать контент и делиться } \\
\text { его содержимым с другими }\end{array}$ \\
\hline $\begin{array}{l}\text { Общение происходит в основном в одном } \\
\text { направлении: преподаватель } \Rightarrow \text { студент }\end{array}$ & Общение происходит в обоих направлениях \\
\hline $\begin{array}{l}\text { Студент - получатель знаний. Его критическое } \\
\text { осмысление полученных знаний не } \\
\text { стимулируется }\end{array}$ & 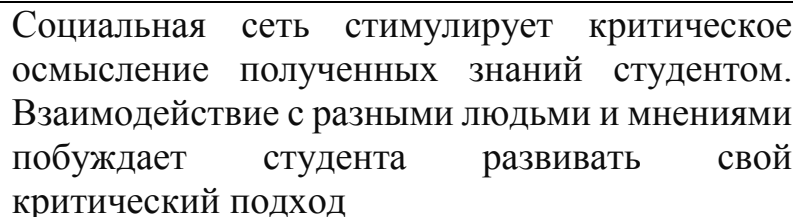 \\
\hline
\end{tabular}

Использование методов кластеризации социальных сетей для персонализации образовательного контента

Для того чтобы делать определенные выводы о влиянии социальных сетей в образовании, моделировать взаимодействие обучаемых и прогнозировать их поведение, необходимо анализировать существующую в них информацию. Анализ данных из социальных сетей состоит из следующих этапов:

- сбор данных;

- очистка данных;

- предварительная обработка данных;

- отслеживание изменений пользовательских данных;

- выбор метода для проведения анализа данных;

- построение графовых структур;

- визуализация данных.

Ясно, что объем информации, циркулирующей в социальной сети, огромный. Поэтому необходимы очистка (отсечение ненужной информации) и предварительная обработка информации (приведение информации к форме, удобной для ее анализа). В результате очистки данных из нее выделяем информацию, представляющую интерес в соответствии с поставленными целями и ее дальнейшей обработкой.

Информация, которую можно извлечь из социальной сети для анализа взаимодействий между пользователями (в данном случае между студентами, преподавателями и студентами, инструкторами и студентами и т.д.), может быть следующая:

- имя пользователя;

- количество подписчиков данного пользователя (количество пользователей, читающих его сообщения);

- количество друзей данного пользователя;

- количество сообщений, посылаемых данным пользователем другому пользователю;

- количество пересылок сообщения данным пользователем;

- внешние ссылки, встречающиеся в сообщениях данного пользователя;

- количество упоминаний данного пользователя другим пользователем;

- количество упоминаний некоторой внешней ссылки данным пользователем; 
- количество просмотров конкретной записи данного пользователя другими пользователями;

- количество лайков (одобрений) конкретной записи данного пользователя.

Одним из направлений анализа социальных сетей является изучение ее структуры, где все участники сети (в данном случае студенты, преподаватели и инструкторы) рассматриваются как вершины графа, соединенные ребрами с другими участниками сети. Этот метод используется для моделирования коммуникационных связей между пользователями сети, анализа потоков распространения информации и нахождения сообществ в сети. Здесь основное внимание уделяется таким характеристикам узлов сети, как центральность, взаимное расположение, интенсивность (вес ребер) и направленность взаимодействий, коэффициент кластеризации (мера связности узлов сети) [11].

Визуализированные графы позволяют выявить узлы, являющиеся ближайшими, найти плотные кластеры активности. Одним из основных параметров графа является «центральность по степени», выражающая отношение количества связей определенного узла к общему количеству других узлов. Если для некоторого узла этот параметр равен 1, это означает, что этот узел связан со всеми остальными узлами сети, если он равен 0, то этот узел является изолированным. Этот показатель показывает степень «знаменитости» узла, показывает, что студент оказывает большое влияние на остальных. На рисунке 2 показан фрагмент визуализированного графа для анализа взаимодействия учащихся в социальной сети. Из рисунка 2 видно, что пользователь сети R1 обладает более высокой степенью центральности, чем пользователь R3 (из R3 отходит меньше линий связи, чем из узла R1).

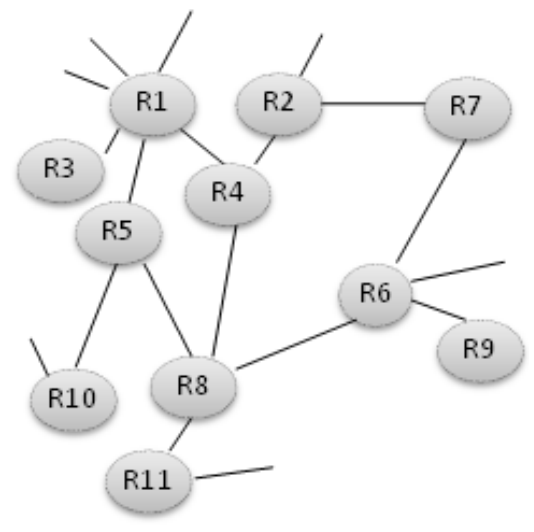

Рис.2. Фрагмент визуализации взаимодействия студентов в социальной сети

\section{Применение $k$-means для кластеризации учебной информации, распространяемой студентами в социальной сети}

В социальных сетях связи могут быть сильными или слабыми в зависимости от интенсивности и частоты общения. И чтобы выбрать нужную информацию из большого объема разнородных данных, необходимо использовать методы, позволяющие анализировать самое важное. Например, можно уменьшить число просматриваемых пользователей, ограничиваясь сравнительно популярными (лидеры) из них, которые формируют мнение большинства. У лидера мнений большое количество подписчиков (друзей), созданных сообщений, лайков и комментариев, от него информация распространяется быстрее и он воздействует на большее количество пользователей.

По мнению некоторых авторов $[12,13]$, совокупный анализ структуры социальных графов и текстовых данных, получаемых из социальных сетей, является наиболее эффективным методом исследования взаимодействий между пользователями сети. Для анализа структуры и содержания информации, которой обмениваются студенты вуза в социальной сети, можно использовать различные методы кластеризации данных: $k$-means и 
c-means, бинарное дерево кластеров и ряд других $[14,15]$.

В нашей статье для кластеризации учебной информации, распространяемой студентами в социальной сети, используется метод k-means (к-средних), являющийся среднеквадратичным отклонением суммы расстояний от каждого объекта кластеризации до центра кластера.

Пусть $N$ - количество студентов, зарегистрированных в конкретной социальной сети, обучающихся по родственной специальности на одном и том же курсе высшего учебного заведения, а $\{X\}$ - общее количество учебных материалов по одинаковой тематике, которыми они обмениваются в сети.

Далее через $X_{i}$ обозначим конкретный $i$-ый учебный материал, которым обменивается в сети $N_{i}$-ое количество студентов. Тогда, чтобы сравнить по популярности $X_{i}$ учебный материал, вводим следующий коэффициент:

$$
\kappa_{i}=\frac{N_{i}}{N} .
$$

Чем ближе этот коэффициент будет к 1, тем конкретный учебный материал будет считаться популярным среди студентов, ближе к 0 - непопулярным.

Но очень часто мы не знаем заранее содержание учебного материала и нелегко схожие по содержанию тексты объединить в одну группу. Для сравнения текстов широко используются такие характеристики, как частота и количество используемых ключевых слов в тексте.

Обозначим через $\lambda_{\kappa}^{i}$ - частоту использования $\kappa$-го ключевого слова в некотором $i$-ом учебном материале, а через $\overline{\lambda_{k}}-$ среднюю частоту $\kappa$-го ключевого слова во всех n-документах.

Среднее значение метрики некоторого $\kappa$-го ключевого слова во всех n-документах:

$$
\overline{\lambda_{k}}=\frac{1}{n} \sum_{i=1}^{n} \lambda_{k}^{i}
$$

При сравнении двух текстов одинаковой длины частоты использования некоторого ключевого слова должны не намного отличаться.

Среднеквадратичное отклонение $\kappa$-го ключевого слова находим как:

$$
\sigma_{k}=\frac{1}{n} \sum_{i=1}^{n}\left(\lambda_{k}^{i}-\overline{\lambda_{k}}\right)^{2}
$$

К одной группе будем относить учебные материалы, в которых среднеквадратичное отклонение частот встречаемости $\mathrm{k}$ ключевых слов находится в некотором допустимом диапазоне, т.е. плотность кластерного ядра ниже некоторого значения.

Кластерным ядром, в общем смысле, называется часть элементов кластера, наиболее приближенная к центру. Предполагается, что в этой области сосредоточено большинство данных кластера.

Радиус кластерного ядра из неравенства Чебышева $[16,17]$ определяется как:

$$
R_{i}=\bar{r}_{i}+\sqrt{2} * \sigma_{i} \text {. }
$$

Здесь $\bar{r}_{i}$ - среднее расстояние между центром кластера и кластерными элементами, а $\sigma_{i}$ - среднеквадратичное отклонение. По меньшей мере $50 \%$ частот ключевых слов учебных материалов удалено не более чем на $\sqrt{2}$ среднеквадратичных отклонений от их среднего значения. 
Плотность кластера рассчитывается следующим образом:

$$
\rho_{i}=\frac{R_{i}}{\overline{M_{i}}}
$$

где $R_{i}$ - радиус кластерного ядра, $\overline{M_{i}}$ - число элементов, составляющих ядро.

Коэффициентом кластеризации учебных материалов будет являться средняя плотность:

$$
\bar{\rho}=\frac{\rho_{1}+\ldots+\rho_{m}}{m} .
$$

Коэффициент кластеризации является мерой того, насколько хорошо связаны узлы графа с конкретным узлом. Когда коэффициент кластеризации ближе к заданному значению - это означает, что граф плотно сгруппирован вокруг некоторых узлов; когда он намного меньше заданного значения - это значит, что связи в графе относительно равномерно распространены среди других узлов. В найденных кластерах сосредоточены узлы графа, которые отражают плотные связи между студентами социальной сети, обменивающимися однотипным по содержанию учебным контентом. В результате проведенного исследования можно выявить, какие учебные материалы интересны студентам, а какие для них не представляют особой ценности. Полученная из социальных сетей информация может быть использована преподавателями высшего учебного заведения для улучшения содержания учебных материалов и их адаптации под индивидуальные особенности студентов.

\section{Заключение}

Таким образом, увлечения человека, представленные в виде графа учебных материалов, обмениваемых студентами в некоторой социальной сети, обеспечивают средства для дальнейшей персонализации учебного контента. На основе анализа информации, полученной из сети, педагоги и инструкторы высшего учебного заведения имеют представления об интересах и предпочтениях как отдельных учащихся, так и групп обучаемых. Эта информация дает преподавателям возможность совершенствовать содержание электронного курса и настраивать его под индивидуальные запросы обучаемого.

\section{Литература}

1. Стародубцев В.А. Персонализация виртуальной образовательной среды // Педагогическое Образование в России, 2015, №7, с.24-29.

2. Harmelen M. Personal Learning Environments / Proceedings of the Sixth IEEE International Conference on Advanced Learning Technologies, USA, pp.815-816.

3. Bennett S., Bishop A., Dalgarno B., Waycott J., Kennedy G. Implementing Web 2.0 technologies in higher education: A collective case study // Computers \& Education, 2012, pp.724-729.

4. Pursel B.K., Xie H. Patterns and Pedagogy: Exploring Student Blog Use in Higher Education // Contemporary Educational Technology, 2014, 5(2), pp.96-109.

5. Schroeder A., Minocha S., Schneider C. The strengths, weaknesses, opportunities and threats of using social software in higher and further education teaching and learning // Journal of Computer Assisted Learning, 2010, pp.159-174.

6. Hosny M. I., Fatima Sh. Facebok in Education: Students, Teachers, and Library Perspectives // journal of Computing, vol.4, №6, 2012, pp.78-86.

7. McLoughlin C., Lee M. Personalised and self-regulated learning in the Web 2.0 era: International exemplars of innovative pedagogy using social software // Australasian Journal of Educational Technology 26(1), March 2010, pp.28-43. 
8. Allen M. An Education in Facebook // Digital Culture and Education, 4(3), 2012, pp.213-225.

9. Pursel K., Hui Xie. Patterns and Pedagogy: Exploring Student Blog Use in Higher Education // Contemporary educational technology, 2014, 5(2), pp.96-109.

10. Ribeiro F.M.S. Contributions of Online Social Networks for E-Learning (Dissertation), Department of Informatics University of Beira Interior, Portugal, 2010, p.70 (On-line). https://ubibliorum.ubi.pt/bitstream/10400.6/3715/1/Contributions of Online Social Networks for E-Learning.pdf

11. Носов В.И. Элементы теории графов, Новосибирск: СГУТИ, 2008, с.107.

12. Charu C. Aggarwal Social Network Data Analytics. Boston: Kluwer Academic Publisher, 2011. 520 p.

13. Батура Т.В. Методы анализа компьютерных социальных сетей // Вестник Новосибирского государственного университета. Серия: Информационные технологии. 2012, т.10, вып.4, с.13-28.

14. Russell M.A. Mining the Social Web, O’Reilly Media, 2011, p.411.

15. Bonatoa A. \& Hadib N. \& Hornc P. \& Prałatd P. \& Wange C. Models of Online Social Networks // Internet Mathematics, 6(3), 2009, pp.285-313.

16. Медведев Г.А. Теория вероятностей и математическая статистика для экономистов; учебник и практикум для бакалавриата и магистратуры, М.: Издательство Юрапт, 2016, c. 284.

17. Mishra N., Schreiber R., Stanton I. and Robert E. Tarjan. Finding Strongly Knit Clusters in Social Networks // Internet Mathematics, 5(1-2), 2008, pp.155-174.

\section{UOT 004.89}

Məmmədova Gülarə A., Ağayev Firudin T., Zeynalova Lalə A.

AMEA İnformasiya Texnologiyaları İnstitutu, Bakı, Azərbaycan

depart10@iit.ab.az

\section{Sosial şəbəkələrin istifadəsi əsasında elektron təhsilin fərdiləşdirilməsi məsələləri}

Hal-hazırda müasir sosial texnologiyalar yüz milyonlarla istifadəçi tərəfindən istifadə olunur, pulsuz, cəlbedici və maraqlıdır. Məqalədə ali təhsil müəssisəsinin elektron təhsilini yaxşılaşdırmaq üçün sosial şəbəkələrdən istifadə imkanları araşdırılır. Sosial şəbəkədə universitet tələbələri arasında yayılan informasıyanın həcminin böyük olmasını nəzərə alaraq, tədris materiallarının kontentinin fərdiləşdirilməsi üçün məqalədə verilənlərin klasterizasiyası metodlarından ( $k$-means) istifadə edilməsi təklif olunur. Tədqiqatın nəticələri ali təhsil müəssisələrinin müəllimləri və instruktorlar tərəfindən e-kurs məzmununun yaxşılaşdırılması və elektron təhsilin fərdiləşdirməsi üçün istifadə edilə bilər.

Açar sözlor: sosial şəbəkə, elektron təhsil, tədris kontentinin fardilaşdirilməsi, klasterizasiya metodu.

\section{Gulara A. Mammadova, Firudin T. Aghayev, Lala A. Zeynalova}

Institute of Information Technology of ANAS, Baku, Azerbaijan

depart10@iit.ab.az

\section{The use of social networks for personalization of electronic education}

Currently, modern social technologies used by hundreds of millions of users are attractive and interesting and available free of charge. The article discusses the possibility of using social networks to improve e-education at higher education institutions. Considering a large amount of information disseminated by university students in the social network, the article proposes the use of data clustering methods - $k$-means to personalize the content of educational materials. The results of the research can be used by teachers and instructors of higher educational institutions to improve the content of e-course and to personalize e-education.

Keywords: social network, e-education, personalized learning content, clustering methods. 